\title{
Degradación de la Fuerza de los Elásticos Intermaxilares de Látex y no Látex
}

\author{
Force Degradation of Latex and Non Latex Intermaxillary Elastics
}

\author{
Mary Loly Farfán Rodriguez'; Manuel Antonio Mattos-Vela² \& Luciano Carlos Soldevilla Galarza3
}

FARFÁN, R. M. L.; MATTOS-VELA, M. A. \& SOLDEVILLA, G. L. C. Degradación de la fuerza de los elásticos intermaxilares de látex y no látex. Int. J. Odontostomat., 11(3):363-368, 2017.

RESUMEN: El propósito del presente estudio fue comparar la degradación de la fuerza entre los elásticos de látex y no látex de 3/16" y 6 oz. La muestra consistió en 30 elásticos por grupo e intervalo de tiempo haciendo un total de 180 elásticos de látex y 180 de no látex. Se midió la fuerza inicial de 30 elásticos de látex y 30 de no látex. Los demás fueron sometidos a tracción estática bajo condiciones orales de humedad y temperatura por 1, 3, 6, 12 y 24 horas antes de la medición de la fuerza con un dinamómetro (Correx $250 \mathrm{~g}$, Alemania). Se emplearon las pruebas de Friedman, Wilcoxon y la U de Mann-Whitney para determinar si existían diferencias significativas. Se encontró que los elásticos de látex presentaron una degradación media de la fuerza de 13,8 \%, 17,4 \%, 18,2 \%, $21 \%$ y 23,4 \% a las 1, 3, 6, 12 y 24 horas, respectivamente. Los elásticos de no látex presentaron una degradación media de la fuerza de 32,5 \%, 39,6 \%, 44,4 \%, 51,1 \% y 56\% a las 1, 3, 6, 12 y 24 horas, respectivamente. Cuando se compararon los valores hallados entre los dos tipos de elásticos en todos los momentos evaluados se encontraron diferencias estadísticamente significativas ( $p<0,001)$. Conclusión: La degradación de la fuerza para los elásticos de látex fue menor al de los elásticos de no látex en todos los intervalos de tiempo.

PALABRAS CLAVE: ortodoncia, látex, aparatos ortodóncicos, módulo de elasticidad

\section{INTRODUCCIÓN}

Los tratamientos ortodónticos correctivos buscan el movimiento dental a una posición adecuada a través de fuerzas mecánicas a los dientes. Los elásticos son fuente de fuerza y se utilizan como componentes activos de la terapia de ortodoncia desde hace algunas décadas. Han sido un valioso complemento de cualquier tratamiento de ortodoncia ya que pueden ser usados en varias configuraciones para la corrección de una maloclusión en particular (Singh et al., 2012). Fueron inicialmente hechos de caucho y luego diseñados en formas sintéticas sin látex (Newgman, 2010; Polur \& Peck, 2010).

El látex natural es un polímero de alto peso molecular que necesita ser procesado para mejorar sus propiedades. El proceso al que es sometido añade productos químicos que pueden causar hipersensibilidad en algunos pacientes y odontólogos. Aproxi- madamente del 0,12 al $6 \%$ de la población y el $6,2 \%$ de los odontólogos son alérgicos al látex (López et al., 2011). Por esta razón es necesario el uso de elásticos sin látex en algunos casos.

Todos los materiales elastómeros sufren fatiga y el medio oral puede influir reduciendo su eficacia al actuar como un agente en la degradación de la fuerza, ya que modifica la estructura molecular de los elásticos debido a daños físicos (traumas mecánicos, alteración en la temperatura); y alteraciones químicas entre el material, la saliva y sustancias de la dieta. (Leo et al., 2013).

Los elásticos con látex y sin látex no presentan un comportamiento similar (Kersey et al., 2003) por lo que es necesario evaluar sus propiedades mecánicas. Se ha reportado en la literatura que los elásticos de

\footnotetext{
${ }^{1}$ Cirujano-Dentista. Egresada de la Facultad de Odontología, Universidad Nacional Mayor de San Marcos, Lima, Perú. ${ }^{2}$ Doctor en Estomatología, Docente de la Facultad de Odontología, Universidad Nacional Mayor de San Marcos, Lima, Perú.

${ }^{3}$ Coordinador Posgrado Ortodoncia y Ortopedia Maxilar, Universidad Nacional Mayor de San Marcos, Lima, Perú.
} 
látex en un medio acuoso $u$ oral pierdan entre $10 \%$ y $40 \%$ de su fuerza inicial entre los 30 minutos y 24 horas después de su aplicación, y los elásticos sin látex pierden entre $19 \%$ y $73 \%$ de su fuerza inicial después de 24 horas. (Alavi et al., 2014; Fernandes et al., 2011; López et al., 2011).

Con la finalidad de poder conocer las características de la fuerza de extensión adecuadas para lograr un movimiento dental requerido, el presente estudio tuvo como objetivo evaluar y comparar la degradación de la fuerza de los elásticos ortodónticos con látex y sin látex en un intervalo de 24 horas sometidos a tracción estática.

\section{MATERIAL Y MÉTODO}

Estudio de tipo experimental in vitro, longitudinal y comparativo. La muestra estuvo conformada por 180 elásticos intermaxilares de látex y 180 de no látex de $3 / 16$ " y 6 onzas de la marca Ortho Classic. Se tomaron como criterios de inclusión paquetes de elásticos que se encontraron antes de la fecha de caducidad, sellados y almacenados en un lugar fresco y oscuro. Todos los elásticos pertenecían a la misma marca comercial y presentaban similar fecha de fabricación. Se realizó una medición de la fuerza inicial de 30 elásticos de látex y 30 de no látex. El resto de elásticos fueron colocados en un medio que simuló las condiciones orales de humedad y temperatura. Este estuvo conformado por un Equipo de baño maría WNB 10 (Memmert, Alemania) que permitía la visualización y control de los valores de temperatura de forma digital, al cual se le agregó agua destilada y la mantenía a 37 ${ }^{\circ} \mathrm{C}$ con una precisión de $+0,25^{\circ} \mathrm{C}$.

Se colocaron pines sobre un tablero de acrílico, separados $14 \mathrm{~mm}$ de distancia unos de otros, lo que representa la longitud del elástico 3/16 estirado tres veces su diámetro.

Los elásticos fueron montados sobre los pines a intervalos de 1 min y permanecieron estirados 14 $\mathrm{mm}$ por 1, 3, 6, 12 y 24 horas en el Equipo de baño maría WNB 10 (Memmert, Alemania) antes de la lectura de fuerza (Fig. 1), la cual fue medida con un dinamómetro de precisión de $250 \mathrm{~g}$ (Correx, Suiza) en la misma secuencia como fueron colocados y con el mismo intervalo de tiempo (Fig. 2).

Los datos de fuerza, tiempo y tipo de elástico fueron registrados en una ficha numerada. El procesamiento y análisis de la información se realizó en el programa estadístico SPSS v.21. Primero se aplicó la Prueba de Kolmogorov-Smirnov para determinar si los datos presentaban una distribución normal. Se empleó la Prueba de Friedman para determinar si había diferencias significativas entre los porcentajes de fuerza perdida dentro de cada grupo y luego la Prueba de Wilcoxon para determinar entre qué intervalos de tiempo existían diferencias significativas realizando una comparación por pares. Finalmente se empleó la prueba U de Mann-

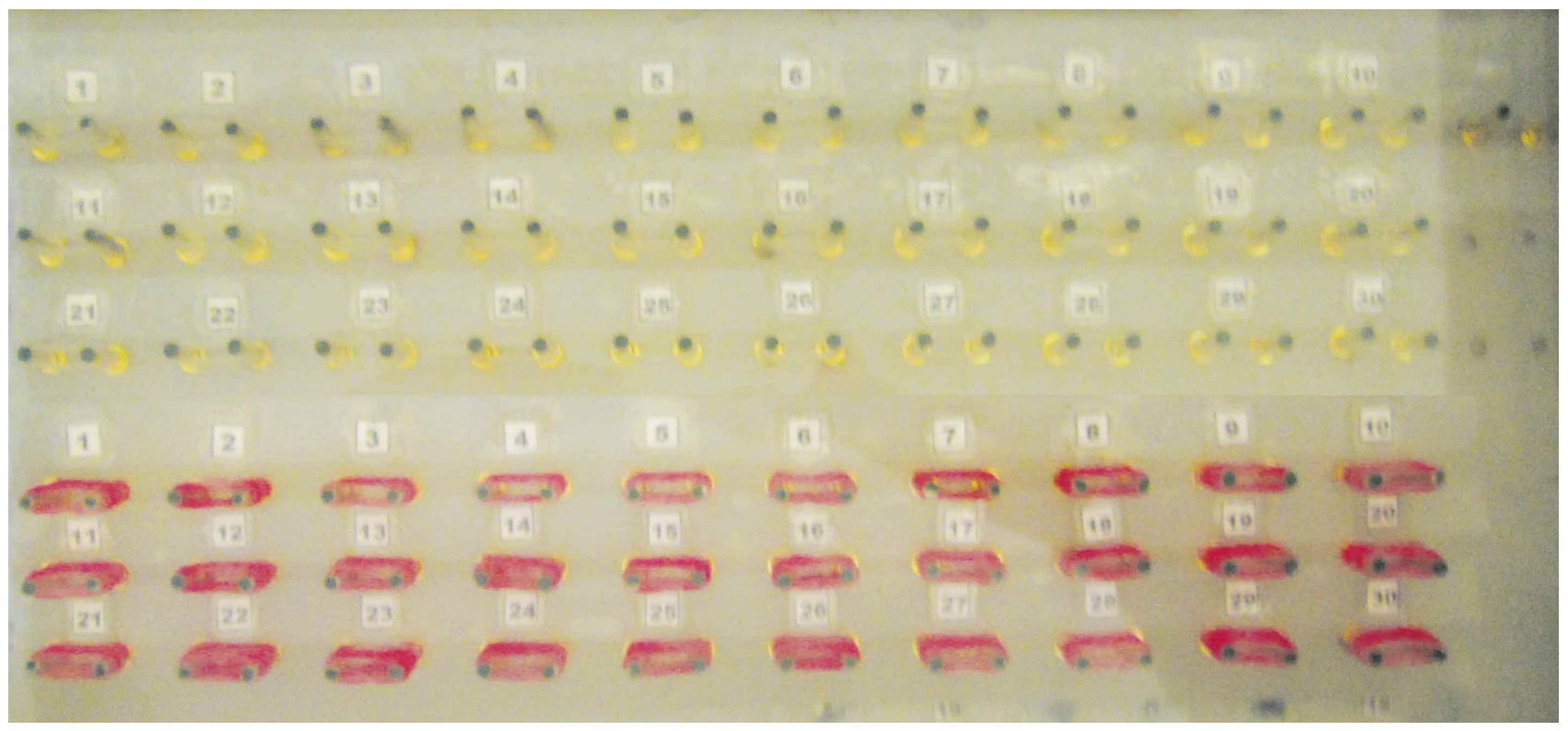

Fig. 1. Ubicación de los 150 elásticos de látex y 150 de no látex en los pines correspondientes dentro de la máquina de baño maría. 
Whitney para comparar los valores entre los grupos de látex y no látex en los distintos intervalos de tiempo. Todas las pruebas estadísticas fueron realizadas con un nivel de significancia del $5 \%$.

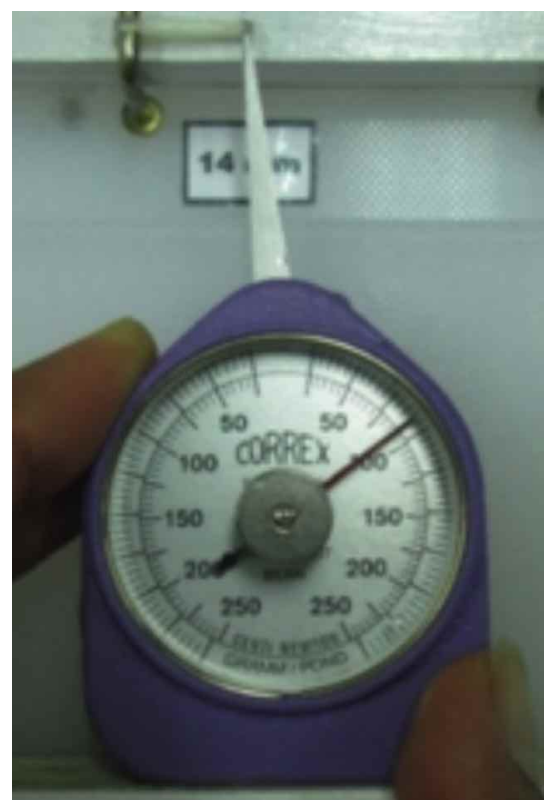

Fig. 2. Medición de la fuerza presentada por los elásticos.

\section{RESULTADOS}

Los elásticos de látex presentaron una disminución media del 13,8 $+/-3,2 \%$ de su fuerza inicial durante la primera hora, $17,4+/-3,3 \%$ a las 3 horas, $18,2+/-3,6 \%$ a las 6 horas, $21+/-3,2 \%$ a las 12 horas y $23,4+/$ $-2,7 \%$ a las 24 horas después de ser sometidos a tracción estática bajo condiciones orales de humedad y temperatura. Los elásticos no látex presentaron una disminución media del $32,5 \%$ de su fuerza inicial durante la primera hora, 39,6 \% a las 3 horas, $44,4 \%$ a las 6 horas, $51,1 \%$ a las 12 horas y $56 \%$ a las 24 horas después de ser sometidos a tracción estática bajo condiciones orales de humedad y temperatura (Tabla I).

Se aplicó la Prueba de Friedman para determinar si había diferencias entre los porcentajes de fuerza perdida dentro de cada grupo. Se encontró diferencias estadísticamente significativas tanto en los elásticos de látex $(p<0,001)$ como en los elásticos no látex $(p<0,001)$ (Tabla I).

Se determinó entre qué intervalos de tiempo existían diferencias significativas dentro de cada grupo mediante la Prueba de Wilcoxon realizando una comparación por pares donde se encontró que en los elásticos de látex existían diferencias estadísticamente significativas entre todos los intervalos de tiempo, excepto entre la tercera y sexta hora donde las medias de la fuerza perdida, $17,4 \%$ y $18,2 \%$ respectivamente no presentaron diferencias significativas $(p=0,263)$. Los elásticos no látex presentaron diferencias estadísticamente significativas entre todos los intervalos de tiempo $(p<0,001)$ (Tabla I).

Tabla I. Valores de fuerza perdida (\%) en los grupos de látex y no látex.

\begin{tabular}{|c|c|c|c|c|c|c|c|}
\hline & & & Fuerza pe & a (\%) & & & \\
\hline Grupo & Tiempo & $\mathbf{n}$ & Mínimo & Máximo & Media* & $\mathbf{D E}^{\dagger}$ & Valor $p$ \\
\hline & $1 \mathrm{~h}$ & 30 & 8,9 & 22,6 & $13,8^{a}$ & 3,2 & \\
\hline & $3 \mathrm{~h}$ & 30 & 10,7 & 23,3 & $17,4^{b}$ & 3,3 & \\
\hline Látex & $6 \mathrm{~h}$ & 30 & 6,9 & 24,2 & $18,2^{b}$ & 3,6 & $<0,001$ \\
\hline & $12 \mathrm{~h}$ & 30 & 15,6 & 29,3 & $21^{\mathrm{c}}$ & 3,2 & \\
\hline & $24 \mathrm{~h}$ & 30 & 17,9 & 27,6 & $23,4^{d}$ & 2,7 & \\
\hline & $1 \mathrm{~h}$ & 30 & 29,4 & 36,2 & $32,5^{\mathrm{a}}$ & 2 & \\
\hline & $3 \mathrm{~h}$ & 30 & 34,9 & 43,5 & $39,6^{b}$ & 1,9 & \\
\hline No lát ex & $6 \mathrm{~h}$ & 30 & 39,4 & 48,6 & $44,4^{c}$ & 1,8 & $<0,001$ \\
\hline & $12 \mathrm{~h}$ & 30 & 47,8 & 55,1 & $51,1^{d}$ & 1,7 & \\
\hline & $24 \mathrm{~h}$ & 30 & 51,5 & 59,4 & $56^{\mathrm{e}}$ & 1,7 & \\
\hline
\end{tabular}

*Letras diferentes indican diferencias estadísticamente significativas $(p<0,001) \quad$ †DE= Desviación estándar.

Tabla II. Comparación de la fuerza perdida (\%) entre los grupos de estudio para cada intervalo de tiempo.

\begin{tabular}{cccccc}
\hline & \multicolumn{2}{c}{ Látex } & \multicolumn{2}{c}{ No látex } & \\
Tiempo & $\mathbf{n}$ & Media (\%) & $\mathbf{n}$ & Media (\%) & Valor $\mathbf{p}$ \\
\hline $1 \mathrm{~h}$ & 30 & 13,8 & 30 & 32,5 & $<0,001$ \\
$3 \mathrm{~h}$ & 30 & 17,4 & 30 & 39,6 & $<0,001$ \\
$6 \mathrm{~h}$ & 30 & 18,2 & 30 & 44,4 & $<0,001$ \\
$12 \mathrm{~h}$ & 30 & 21 & 30 & 51,1 & $<0,001$ \\
$24 \mathrm{~h}$ & 30 & 23,4 & 30 & 56 & $<0,001$ \\
\hline
\end{tabular}




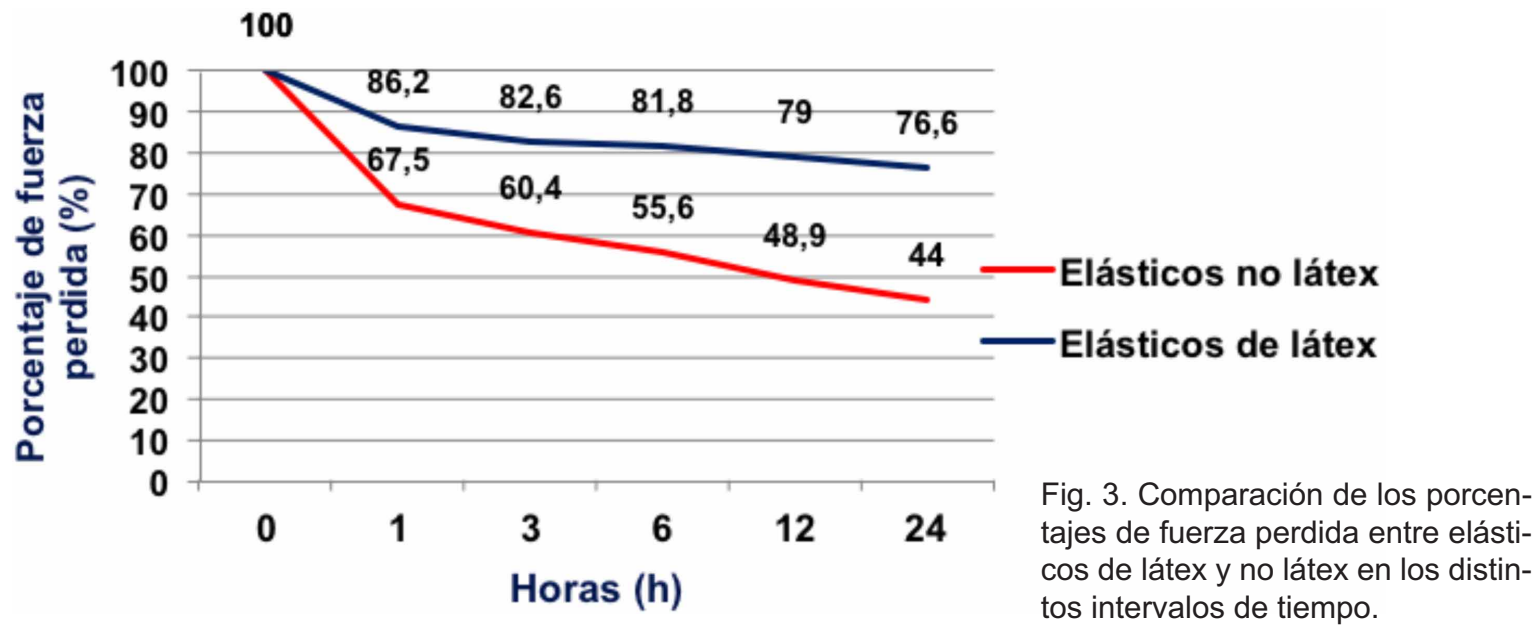

Se compararon las medias de los porcentajes de fuerza perdida en cada intervalo de tiempo entre los elásticos de látex y no látex por medio de la prueba $U$ de Mann-Whitney y se encontraron diferencias estadísticamente significativas en todos los intervalos de tiempo $(p<0,001)$ (Tabla II). Los elásticos no látex presentaron un mayor porcentaje de fuerza perdida al compararlos con los elásticos de látex (Fig. 3).

\section{DISCUSIÓN}

El medio oral actúa como un agente en la degradación de la fuerza de los elásticos de látex y no látex, ya que modifica su estructura molecular interna, ya sea por factores como el medio húmedo, fluctuaciones de temperatura y $\mathrm{pH}$, sustancias de la dieta, acción enzimática y microbiana u otros factores indefinidos. Por tal motivo, el presente estudio trató de reproducir algunas variables como humedad, temperatura, extensión y tiempo de uso de los elásticos, in vitro, de tal manera que estas condiciones pudieron ser controladas con precisión. Los resultados obtenidos coincidieron con los hallazgos de otros estudios donde también encontraron que las fuerzas iniciales fueron menores en los elásticos de látex (Kersey et al.) y mayores en las de no látex (Alavi et al.; Russel et al., 2001; López et al.) que las especificadas por el fabricante. Sin embargo, también se encontraron resultados opuestos, donde las fuerzas iniciales fueron mayores en los elásticos de látex (López et al.; Russel et al.) y menores en las de no látex (Kersey et al.) que las especificadas por el fabricante. Esto demuestra la variación de la fuerza inicial de los elásticos que puede ser atribuida a las características que presentan de acuerdo a cada fabricante.
Algunos estudios in vitro se han realizado con un modelo experimental dinámico en el que se simula los movimientos de apertura y cierre de la boca para estirar los elásticos, sin embargo, Kersey et al. declararon que el repetitivo estiramiento de los elásticos provoca una pérdida de la fuerza mayor en comparación con la prueba estática, pero después de una hora la pérdida es similar en ambas pruebas. Liu et al. (1993) mostraron que después de 200 ciclos de estiramiento no habían diferencias significativas en la degradación de la fuerza de los elásticos. Por lo tanto, para el presente estudio se eligió un modelo experimental estático.

Las comparaciones se realizaron mediante el uso del porcentaje de degradación de la fuerza en lugar de la fuerza real generada debido al gran número de extensiones evaluadas por diferentes autores.

Al igual que otros estudios (Kersey et al.; Fernandes et al.; Wang et al., 2007; Gioka et al., 2006) la mayor degradación de la fuerza se produjo durante la primera hora, $13,8 \%$ para los elásticos de látex y $32,5 \%$ para los elásticos no látex, y se hizo más lenta con el pasar del tiempo. Los elásticos de látex presentaron una degradación media de la fuerza de $23,4 \%$ a las 24 horas. Este resultado coincide con los de Gioka et al. donde se obtuvo una disminución del $23,02 \%$ de la fuerza a las 24 horas en elásticos de $3 / 16$ " y 6,5 oz de la marca Ortho Technology. Fernandes et al. encontraron una variabilidad en la disminución de la fuerza, entre el $10,6 \%$ y el $31 \%$ a las 24 horas, con una medida media de $15,13 \%$ en la marca Morelli, 21,16 $\%$ en American Orthodontics y $16,67 \%$ en TP.

Los elásticos no látex presentaron una degradación media de la fuerza del 56 \% a las 24 horas. 
Alavi et al. encontraron una disminución del 4 al 7,5\% después de 1 hora y entre $19 \%$ y $38 \%$ a las 24 horas. Kersey et al. reportaron una disminución del $31 \%$ de la fuerza en un estudio estático y el $47 \%$ en un estudio dinámico. Russell et al. reportaron de $15 \%$ a $20 \%$ en la primera hora y de $20 \%$ a $43 \%$ a las 24 horas. En otro estudio mencionado por Lopez et al. se encontró que la fuerza perdida fue del $24 \%$ en medio seco y $73 \%$ en medio húmedo después de 24 horas.

Cuando se comparó la degradación de la fuerza entre los elásticos de látex y no látex se encontraron diferencias significativas en todos los intervalos de tiempo. Estos resultados coinciden con los de Kersey et al. donde al evaluar elásticos de 14" y 4.5 oz marca América Orthodontics encontraron que los elásticos de látex mantuvieron fuerzas más altas en el tiempo perdiéndose el $17 \%$ de su fuerza inicial, mientras que los elásticos no látex perdieron el $31 \%$ en 24 horas. Russell et al. encontraron diferencias entre los elásticos de látex y no látex de la marca GAC, pero no en los de la marca Masel. De igual manera López et al. al comparar elásticos de látex y no látex de 1/4" y 4 oz no encontraron diferencias significativas entre las fuerzas iniciales, a las 8 y 24 horas en las marcas GAC y LANCER en condiciones húmedas. Existe una gran variabilidad en los resultados al comparar elásticos de látex y no látex, que puede ser atribuida al tipo de elástico, al fabricantey a los diferentes entornos experimentales. Ningún producto de la marca Ortho Classic se ha puesto a prueba en los estudios citados anteriormente, por lo que las comparaciones directas son limitadas.

No hay un consenso en la literatura sobre qué cantidad de degradación de la fuerza es importante ya que est o depende de la magnitud de la fuerza deseada por el clínico. Algunos autores han utilizado una diferencia del $10 \%$ como un valor clínicamente significativo (Kersey et al.).

En la práctica clínica real los elásticos son expuestos a numerosos factores intraorales, por lo tanto, no se pueden tomar decisiones clínicas basadas en estudios in vitro. Sin embargo, este tipo de estudio es útil como guía para posibles experimentos clínicos en el futuro y puede contribuir a mejorar el conocimiento del comportamiento mecánico de los elásticos intermaxilares lo que permite una mayor previsibilidad en su aplicación.

Los autores del presente estudio comparten la idea de Kersey et al. y López et al. que debido a la variabilidad de resultados en los estudios, es aconsejable que los profesionales pongan a prueba una muestra de sus elásticos antes de usarlos o de adquirir grandes cantidades para asegurarse que los niveles de fuerza producidos caen dentro del rango esperado según lo especificado por el fabricante.

En todos los intervalos de tiempo se encontró una significativa degradación de la fuerza tanto en los elásticos de látex y no látex, excepto entre la tercera y sexta hora para los elásticos de látex.

Existen diferencias en el porcentaje de degradación de la fuerza en todos los intervalos de tiempo entre los elásticos de látex y no látex, siendo este porcentaje menor en los elásticos de látex.

Debido a las altas tasas de pérdida de fuerza en los elásticos no látex, es importante que estos se cambien a intervalos regulares no superiores a 3-4 horas.

FARFÁN, R. M. L.; MATTOS-VELA, M. A. \& SOLDEVILLA, G. L. C. Force degradation of latex and non latex intermaxillary elastics. Int. J. Odontostomat., 11(3):363-368, 2017.

ABSTRACT: The purpose of this study was to compare the force degradation between latex and non latex elastic $3 / 16$ " 6oz. The sample consisted of 30 elastics per group and time interval, 180 latex and 180 non latex elastics were used. The initial force was measured in 30 latex elastics and 30 non latex elastics. Others elastics were subjected to static traction in conditions of humidity and temperature for 1, 3, 6, 12 and 24 hours. The force was measured with a dynamometer (Correx $250 \mathrm{~g}$, Germany). Wilcoxon test and Mann-Whitney test was employed to determine significant differences. The latex elastics showed a mean force degradation of $13.8 \%$ during the first hour, $17.4 \%$ at 3 hours, $18.2 \%$ at 6 hours, $21 \%$ at 12 hours and $23.4 \%$ at 24 hours. The non elastics latex showed a mean force degradation of $32.5 \%$ during the first hour, $39.6 \%$ at 3 hours, $44.4 \%$ at 6 hours, $51.1 \%$ at 12 hours and $56 \%$ at 24 hours. It was concluded that the latex elastics force degradation was less than the non-elastics latex in all times.

KEY WORDS: Orthodontics; Latex; Orthodonctic Appliances, Elastic Modulus

\section{REFERENCIAS BIBLIOGRÁFICAS}

Alavi, S.; Rahnama, A.; Hajizadeh, F. \& Ardekani, H. An In-vitro comparison of force loss of orthodontic non-latex elastics. J. Dent. (Tehran), 11(1):10-6, 2014. 
Andreasen, G. \& Bishara, S. Comparison of alastik chains with elastics involved with intra-arch molar to molar forces. Angle Orthod., 40(3):151-54, 1970.

Beltri, P.; Bartolome, B.; Torres, L. \& Planells, P. La alergia al látex en la consulta dental. Cient. Dent., 2(2):133-9, 2005.

Bishara, S. \& Andreasen, G. A comparison of time related forces between plastic alastiks and latex elastics. Angle Orthod., 40(4):319-28, 1970.

Brantley, W.; Salander, S.; Myers, C. \& Winders, R. Effects of prestretching on force degradation characteristics of plastic modules. Angle Orthod., 49(1):37-43, 1979.

Canut, J. A. Ortodoncia clínica y terapéutica. $2^{\text {nd }}$ Ed. Valencia. Masson, 2000.

Fernandes, D.; Abraho, G.; Elias, C. \& Mendes, A. Force Relaxation Characteristics of Medium Force Orthodontic Latex Elastics: A Pilot Study. ISRN Dentistry., 1(1):1-5, 2011.

Gil, M.; Barriga, F. \& Pérez, J. Alergia al látex en los trabajadores sanitarios Vigilancia de la salud. Med. Segur. Trab., 53(3):1-9, 2007.

Gioka, C.; Zinelis, S.; Eliades, T. \& Eliades, G. Orthodontic latex elastics: a force relaxation study. Angle Orthod., 76(3):475-9, 2006.

Kersey, M.; Glover, K.; Heo, G.; Raboud, D. \& Major, P. A comparison of dynamic and testing of latex and nonlatex orthodontic elastics. Angle Orthod., 73(2):181-6, 2003.

Lacerda dos Santos, R.; Melo, M. \& Vilela, M. The influence of ph levels on mechanical and biological properties of nonlatex and latex elastics. Angle Orthod., 82(4):709-14, 2012.

Langlade, M. Optimización de elásticos ortodóncicos. $1^{\text {a }}$ Ed. Nueva York, GAC international, 2000.

Langlade, M. Terapêutica ortodôntica. $3^{\text {th }}$ Ed. Brasil, Santos, 1985.

Leo, J.; Gallo, D.; Santana, R.; Guariza, O.; Camargo, E. \& Tanaka, O. Influence of different beverages on the force degradation of intermaxillary elastics: an in vitro study. J. Appl. Oral. Sci., 21(2):145-9, 2013

Liu, C.; Wataha, J. \& Craig, R. The effect of repeated stretching on the force decay and compliance of vulcanized cis-polyisoprene orthodontic elastics. Dent. Mater., 9:37-40, 1993.

López, N.; Vicente, A.; Bravo, L.; Calvo, J. \& Cantera, M. In vitro study of force decay of latex and non-latex orthodontic elastics. Eur. J. Orthod., 34(1):202-7,2011.

Loriato, L.B.; Wilson, A.; Pacheco, W. Consideraçôes clínicas e biomecánicas de elásticos em ortodontia. R. Dental Press., 5(1):44-57, 2006.

Martins, M.; Moraes, A.; Oliveira, A.; Andrade, M.; Ferreira, V. \& Sá, $\mathrm{S}$. Estudo comparativo entre as diferentes cores de ligaduras elásticas. R. Dental Press Ortodon. Ortop. Facial., 11(4):81-90, 2006.

Matheu, V.; Rodríguez, A.; Gracia, M.; Sánchez, I.; Pérez, E. \& De Frutos, C. Alergia al látex en el servicio de urgencias y emergencias. Emergencias. 18(1):94-100, 2016.

Newgman, R. Biomêcanica dos elásticos intermaxilares na ortodontia: Clase II e Clase III. Tesis para obtener el título de Especialista en Ortodoncia. Instituto de ciencias de la salud Funorte/Soebras. 2010

Polur, I. \& Peck, S. Orthodontic elastics: Is some tightening needed? Angle Orthod., 80(5):988-989, 2010.

Russel, K.; Milne, A.; Khanna, R. \& Lee, J. In vitro assessment of the mechanical properties of latex and nonlatex orthodontic elastics. Am. J. of Orthod. Dentofacial Orthop., 120(1):36-44, 2001.

Sauget, P.; Stewart, K. \& Katona, T. The effect of pH levels on nonlatex vs latex interarch elastics. Angle Orthod., 81(6):10704, 2011.

Singh, V.; Pokhrael, P.; Pariekh, K.; Roy, D.; Singla, A. \& Biswas, K. Elastics in orthodontics: a review. Health Renaissance, 10(1):49-
56.

Uribe, G.A. Ortodoncia teoría y clínica. $1^{\text {st }}$ Ed. Medellín, Corporación para investigaciones biológicas, 2004.

Wang, T.; Zhou, G.; Tan, X. \& Dong, Y. Evaluation of force degradation characteristics of orthodontic latex elastics in vitro and In vivo. Angle Orthod., 77(4):688-93, 2007.

Wong, A. Orthodontic elastic materials. Angle Orthod., 46(2):196-205, 1976.

Dirección para correspondencia

Mary Loly Farfán Rodriguez

Facultad de Odontología

Universidad Nacional Mayor de San Marcos

Lima

PERÚ

E-mail: maryloly133@hotmail.com

Recibido : 30-06-2016

Aceptado: 26-07-2017 